\title{
Todo el mundo sabe. Difusión y apropiación de las técnicas del yoga en Buenos Aires (Argentina)
}

Mercedes Saizar

Investigadora del Consejo Nacional de Investigaciones Científicas y Técnicas (CONICET) en el Centro Argentino de Etnología Americana (CAEA). Professora Asociada en el Instituto Universitario Nacional del Arte. Doctora en Cultura y Sociedad

Resumo En este trabajo, la autora presenta al yoga como una de las prácticas que permiten el acceso y la iniciación a otras búsquedas espirituales y de salud propias del movimiento de la Nueva Era. Analiza el proceso de apropiación y refiguración de las nociones propias de la disciplina oriental, su aparición y difusión, tomando en cuenta tanto la perspectiva de los practicantes -usuarios y especialistascomo el material proveniente de diversos medios de divulgación masivos, tales como revistas, libros, videos de práctica, programas de televisión y foros de Internet dedicados a la temática.

Palabras claves: difusión; apropiación; yoga; Buenos Aires; Argentina.

\section{Introducción}

$\mathrm{E}$ N LAS ÚlTimas DÉCADAS, el impacto y difusión de las terapias alternativas ha sido motivo de variados estudios en el campo de las ciencias sociales. Analizado desde distintas perspectivas, el fenómeno de la apropiación de estas disciplinas ha sido estudiado considerándolo parte de los movimientos sociales asociados a la Nueva Era (Albanese, 1990 y 1992; Barroso, 1999; Carozzi, 1999); como parte del surgimiento de religiones alternativas provenientes de distintas corrientes espirituales, técnicas holísticas y terapias heterodoxas de orígenes socioculturales diversos (Carini, 2004); como la respuesta a búsquedas de terapias delicadas, de carácter holístico y no invasivo (Douglas, 1998); como una selección frente a la ineficacia de la biomedicina en el tratamiento de las "nuevas enfermedades"(Laplantine, 1999; OMS, 2002); como una selección terapéutica coherente con definiciones totalmente diferentes a las propuestas por el modelo biomédico en lo que respecta a la etiología de la enfermedad, la salud, las desviaciones y el poder terapéutico (Mc Guire y Kantor, 1998); como parte de las ofertas terapéuticas de un sistema etnomédico (Idoyaga Molina, 1997 y 2002; Pitluk, 2002; Saizar, 2005 y 2006); como selecciones terapéuticas reflejo de estilos de vida (Pitluk, 2002), entre otros.

Si bien las perspectivas en juego presentan matices en su abordaje del tema, los distintos autores coinciden en que la presencia de las terapias de Nueva Era o de raigambre oriental es cada vez mayor en el occidente, que su oferta se multiplica y diversifica constantemente y que su uso ha sido incorporado a la dinámica de la atención a la salud.

En esta oportunidad, nos interesa analizar parte de este fenómeno, enfocándonos en el caso del yoga en el área Metropolitana de Buenos 
Aires, puesto que presenta ciertas particularidades que merecen ser consideradas. La primera de ellas es que él fue una de las primeras disciplinas orientales elegidas por individuos enculturados en occidente; la segunda, el impacto y permanencia que la disciplina obtuvo entre individuos de distintos grupos sociales y económicos; la tercera, el creciente uso de sus nociones -aunque ampliamente resignificadas- en la descripción de la etiología y modalidad de terapia de variadas dolencias; y finalmente, el hecho de que la disciplina en cuestión presenta una amplitud de oferta y difusión que implica, entre otras cuestiones, transformaciones semánticas de nociones en torno a la salud y la enfermedad y estrategias de complementariedad con otras medicinas y otros sistemas de creencias vigentes en el área de estudio.

Teniendo en consideración estas cuestiones, delinearemos la aparición y difusión del yoga desde sus inicios hasta la actualidad, prestando especial atención al proceso de resignificación de las nociones propias de la disciplina en su lugar de origen. Para ello, nos basaremos en materiales de distintos tipos: por un lado, utilizaremos fuentes bibliográficas (como libros y revistas de divulgación que se ocuparon específica o parcialmente de la temática en distintas épocas), materiales cedidos por los informantes, tales como videos y libros de divulgación de la disciplina y distintas páginas de Internet en donde consultamos la existencia de sitios dedicados a la temática de las alternativas en general y al yoga en particular. Por otra parte, recurriremos al material proveniente de entrevistas abiertas, extensas y recurrentes a usuarios y especialistas del yoga en el área Metropolitana. Este último material es de fundamental importancia, puesto que nos permitirá conocer las experiencias, ideas y reflexiones en torno a la práctica de la disciplina en nuestra área de estudio, considerando la perspectiva de los actores sociales involucrados, tanto especialistas como usuarios. Dentro de este grupo, las diferencias de edad y de rol nos posibilitan obtener relatos que reflejan distintas experiencias en el acercamiento al yoga. El trabajo de campo se realizó en el período 2006-2007, como parte del desarrollo de una Beca Post Doctoral financiada por el Consejo Nacional de Investigaciones Científicas y Técnicas de la Argentina, entidad a quien agradezco tal posibilidad.

\section{Yoga en Buenos Aires}

El yoga comenzó a practicarse en nuestro país a principios del siglo XX y desde ese momento hasta la actualidad no ha dejado de ampliar su campo de impacto. En el actual contexto de selección de terapias alternativas, él se presenta como una de las disciplinas por la que ciertos individuos transitan al menos una vez en sus caminos en búsqueda de salud. Así, la diversidad de opciones de costo y de modalidad de práctica, la apropiación y aceptación por parte de grupos católicos, la propuesta de un ejercicio físico delicado del cuerpo, la compatibilidad que los usuarios encuentran entre su sistema de creencias y las propuestas de la disciplina en cuestión y, finalmente, la posibilidad de gozar de sus beneficios sin la necesidad de adoptar un nuevo estilo de vida, posicionan a la práctica del yoga como una opción no sólo preferencial, sino de posible iniciación en otras búsquedas asociadas al contexto de la Nueva Era.

En sus inicios tempranos, el yoga era una práctica dirigida a sectores urbanos con altos niveles de educación e ingreso económico, lo que la posicionaba como una disciplina espiritual de elite. Estos grupos, generalmente formados por personas que se conocían con anterioridad, invitaban a especialistas orientales de fama reconocida -Maestros Yoguis-, para que viniesen a Buenos Aires a transmitir los principios básicos de la disciplina y su práctica. Algunos de estos especialistas se quedaron en el país y fundaron escuelas de enseñanza, creando a su vez nuevas cohortes de especialistas que comenzaron a identificar su práctica y los fundamentos de la misma con los lineamientos de su fundador. A modo de ejemplo, Barroso (1999, p. 28) distingue en su obra la importancia fundamental que tanto el Maestro Swami Vivekananda como el Maestro Soyen Shaku tuvieron en el acceso de la cultura oriental a occidente. El impacto de sus obras posibilitó, respectivamente, la instalación de las primeras Sociedades Vedanta y centros de Budismo Zen en los Estados Unidos en los inicios del Siglo XX. Carini (2004) señala la figura de Taisen Deshimaru, maestro japonés, que instalado en Europa creó distintos centros de Budismo Zen y cuyo impacto en la Argentina se mediatizó a través del maestro Estefan Thibaud, antiguo discípulo de Deshimaru. En lo que respecta al yoga, un caso ejemplar es el de la Maestra Yogui Indra Devhi (1900-2002) cuya obra, iniciada a mediados del siglo XX, continúa hasta nuestros días, multiplicándose el número de sedes donde se dicta la práctica del yoga sobre la base de las enseñanzas de su fundadora.

En la India, el yoga forma parte de uno de los seis sistemas filosóficos o darsanas, aceptados por el brahmanismo, contrariamente a lo que sucede con el budismo o el jainismo, que son considerados sistemas heréticos (Eliade, 1999). Como nota el autor, el yoga más reconocido en occidente es el que se desprende de las formulaciones de Patanjali, probablemente debido a su gran difusión y a la existencia de numerosos 
escritos y comentarios a su obra (Tola y Dragonetti, 2006) $)^{1}$. Las diferencias entre unos y otros sistemas se basan en aspectos de suma complejidad que hacen, a grandes trazos, a la unicidad o diversidad del universo, la realidad o irrealidad del mismo y a la existencia de un Absoluto y sus posibles manifestaciones encarnadas en la materia (al respecto, sugerimos leer Zimmer (1973), Eliade (1999) y Masson Ourssel (1962)). Estas distinciones que se encuentran en los libros eruditos no forman parte del conocimiento del yoga que poseen los occidentales en general. Al respecto, es necesario recordar que los procesos sociales de transmisión de una cultura o religión implican una reinvención y, por lo tanto, una diferenciación de la original (Carini, 2004). En el caso de nuestro país, podríamos hablar de una condensación de contenidos, los que, proviniendo de distintas ramas filosóficas y prácticas orientales, se presentan como una totalidad bajo el rótulo de Yoga.

Como notáramos en trabajos anteriores (Saizar, 2005), pueden distinguirse dos tipos de especialistas sobre la base de su formación en la disciplina -autodidacta o sistemática- y la dedicación -exclusiva o complementaria- a la enseñanza y práctica del yoga. A pesar de que en ambos grupos se encuentran considerables diferencias en lo que hace a las reflexiones filosóficas sobre el yoga, encontramos que en nuestra área de estudio las diferencias marcadas por los estudiosos de los sistemas de creencias orientales no encuentran eco en las perspectivas de los especialistas, sean del tipo que sean. A modo de ejemplo, un especialista formal define al yoga como un sistema de pensamiento que encuentra sus bases en los sistemas budistas e hinduistas, con rasgos del brahmanismo y de los vedantas, lo que, al entender de Eliade (1999), sería incorrecto. Podría aventurarse que en el proceso de apropiación de la disciplina y teniendo en cuenta las insalvables diferencias entre sus nociones y las propias de Occidente, los conceptos de distintos sistemas -contradictorios entre sí en Oriente- han sido sumados a una sola tradición que postula la idea de individuo como un mero portador material de una sustancia inmaterial, que es en sí misma eterna, inmutable e imperecedera, parte individual de un Absoluto Universal, que es condenada a encarnar una y otra vez durante miles de años hasta poder despojarse de la sustancia kármica y así conseguir la liberación definitiva, el cese del dolor y de la existencia material.

Al respecto, es interesante considerar el impacto que obtuvo la obra del sacerdote jesuita Ismael Quiles (1906-1993), quien tradujera varios textos sagrados hindúes, adecuándolos a las ideas y prácticas del catolicismo. Su obra, fruto de su estancia en la India en distintos centros religiosos, rescata aquellas nociones de la disciplina oriental que, a su criterio, permitirían a los católicos occidentales acceder a una filosofía que postula la experiencia del cuidado del cuerpo como recipiente de lo sagrado. Quiles (1987) señala que el aporte específico del yoga es la obtención de un mayor desarrollo de ciertas técnicas de concentración mental y el recurso de la utilización del cuerpo para que este contribuya a la realización de la liberación del espíritu, aspecto que se integra, en este marco, a la ascética y a la mística cristiana.

Quiles (1987) sintetizó en varias de sus obras una aproximación al yoga oriental desde una perspectiva católica occidental, creando además una carrera de Estudios Orientales en la Universidad del Salvadorde la cual se desprendió luego la Tecnicatura en Yogaen la cual han sido formados muchos de nuestros informantes. Sus aportes transformaron las ideas clásicas del yoga oriental, apropiándose de ciertos contenidos y resignificando otros que eran contrarios a las nociones católicas, por ejemplo, las ideas asociadas a la rueda de encarnaciones o samsara, en contraposición a la idea de un alma inmortal y única que se corresponde a una existencia material perecedera y única; la noción católica de la resurrección de los cuerpos con sus almas al fin de los tiempos, contraria a la noción hindú de materia perecedera y partícula eterna (prakriti-purusha) pero despojada de materia; la idea de ignorancia en contraposición a la noción católica de la existencia de una noción moral valorativa del mal y del bien, o la noción católica de piedad y comprensión hacia el prójimo, en tanto en Oriente cada encarnación supone un dharma específico, es decir, una serie de conductas culturalmente esperables de acuerdo a la casta y a la evolución lograda, al tiempo que los actos no son moralmente connotados en forma alguna, sino simplemente atribuidos a la ignorancia.

Otro de los factores que ayudaron a la divulgación de la disciplina oriental en Buenos Aires fue el acceso que a ésta tuvieron distintos individuos, signados por una impronta de búsqueda de nuevas terapias y una nueva espiritualidad. En la década del sesenta, se formaron en la ciudad de Buenos Aires una serie de grupos cuyo objetivo era la exploración de nuevas prácticas que permitieran mejorar la calidad de vida, contrarrestando los efectos negativos de una educación considerada rígida y un estilo de vida que percibían como alejado del contacto con "la naturaleza". Estos grupos eran coordinados por mujeres y hombres pertenecientes a grupos sociales de buen ingreso económico y alto nivel de instrucción y concurrían a ellos otros hombres y mujeres de las mismas condiciones. En estos encuentros se trabajaba con una multiplicidad de técnicas de raigambre oriental y occidental, en el contexto de distintos talleres que combinaban prácticas corporales, con prácticas de respiración, meditaciones grupales e individuales y lecturas comunes. Alicia, una integrante de estos grupos formados en los inicios de la década del sesenta, nos decía:

Me conecté con algo que en Buenos Aires hace mucho que comenzó en 1960, que es el grupo que en aquel 
momento dirigía Susana Milberman, Susana Rivara de Milberman, que incorporaba lo que ahí en esa época se necesitaba para sentirse bien y que estaba como que era tabú, no se podía hacer, que quedaba mal, que la sociedad, es decir, expresarse a sí mismo. Entonces había muchos esquemas prefijos en la sociedad: portáte bien, sentate derecha, cruza las piernas... y todas esa serie de cosas y también me acuerdo que Teba, mi maestra, en aquel entonces, porque fue un grupo grande este, que se derivó después en los institutos de Río Abierto, Arroyo, Gimnasia Expresiva Rítmica Yoga, GREY, se llama eso, o sea que mira vos la sigla: Gimnasia, Rítmica, Expresiva, es poder expresar, Yoga, que es trabajo interno. Entonces, allí ellos como que fueron creando este sistema, en el cual, se trabajaba con música, la música tiene ritmos, entonces, la gimnasia era con ritmo, pero en relación con la música. No con un esquema muy prefijo, pero teniendo en cuenta el cuerpo humano. Es decir, que el cuerpo humano tiene articulaciones, tiene músculos, un sistema nervioso, un sistema energético, tiene sistema digestivo orgánico, entonces, todo eso se fue como muy tendiendo en cuenta, y el sistema emocional. O sea que todo eso viene también relacionado con las pautas del yoga, pero no era un yoga estático, era tomar vertientes filosóficas, porque ellas trabajaron mucho con pautas de los griegos, posturas apolíneas, como para salir de la gimnasia "uno, dos, tres, cuatro", que se hacía gimnasia rítmica en los colegios. Esto fue entonces un lindo movimiento acá en Buenos Aires, muy, muy, muy hermoso. Y ya en la década del 60, del 70, poder hacer eso era como un permiso muy grande. Y poder expresar, sobre todo, la expresión venía con el movimiento, también de la parte cardiaca, pectoral, brazos y sonido. Incorporar el sonido en aquel momento era una cosa revolucionaria. Poder expresar cansancio con un suspiro, era totalmente no solamente propuesto, sino ¡qué bueno que lo podías hacer! Inclusive en un principio no lo podías hacer, estabas acostumbrado a reprimir, cómo ibas a expresar cansancio, eso quedaba mal, o que contenta estoy, también. A veces se cantaba, se cantaba no sabiendo cantar, ese también era un permiso, porque ellos descubrieron, no sé, se reunían, un grupo que estaba allí elaborando la cosa, y tenía cada uno sus necesidades y sus comentarios. Había personas, por ejemplo, que les hubiera gustado ser bailarinas, pero no tenían el cuerpo que demandaba la situación, pero sin tener el cuerpo también se podía bailar, se podía expresar, entonces las músicas eran muy importantes. Digamos, como que pronto se fue incorporando todo eso a nivel, que te puedo decir, ejercitación. A nivel de que se pudiese, y bueno, la gente iba, como vos vas a hacer gimnasia, se reunían gente que no se conocían, pero el instructor era el que daba la pauta. Y también esto tuvo una cosa muy buena, que fue desarrollar la parte mental de las personas, entonces había conferencias por ejemplo yo me acuerdo, en aquel momento recién llegaba Swami Shami Prevanandha a la Argentina, que tenía traductor, ni siquiera hablaba en castellano, y este, hicieron una reunión, invitaron a toda la gente, estuvo precioso, y son...eran aperturas que aparecían. Aperturas de otras relaciones. También se proponía poder estudiar filosofía, occidental, que aparecieron profesores de filosofía, de la facultad, muchachos que estaban por recibirse, vino Emilio Comar, en aquel momento era una persona extraordinaria, daba unas conferencias muy buenas sobre el hombre, sobre la sociedad y así esto fue como una apertura.

De estos grupos de práctica y estudio surgieron muchos de los especialistas del yoga que hoy dictan sus propios talleres y forman nuevos especialistas; otros individuos, como es el caso de Alicia, continuaron en un camino de búsqueda espiritual y habiendo pasado por la práctica del yoga, indagaron luego en distintas terapias alternativas, realizando cursos y seminarios en distintos workshops, organizados y dirigidos por especialistas extranjeros o vernáculos. Años más tarde, se especializaron como terapeutas de otras medicinas orientales como el reiki, la acupuntura o el feng shui, e incursionaron en otros casos en modalidades que, si bien nacieron en Occidente, remiten a marcos filosóficos orientales, como el healing touch o la Técnica Alexander. En el relato de sus experiencias, el yoga aparece como una práctica iniciática.

Carozzi (2001) apunta en su estudio sobre la Nueva Era en Buenos Aires, el lugar preponderante que, para los años 80, tuvieron tanto Miguel Grinberg como Juan Carlos Kreimer en la difusión de variadas y renovadas disciplinas alternativas, los que al tiempo que convocaban conferencistas internacionales especialistas en distintas terapias alternativas, formaban grupos de contacto entre los cultores de la Nueva Era y plasmaban este fenómeno en dos revistas de divulgación creciente: Mutantia y Uno Mismo. Carini (2004) señala la creación, durante la década del 90, de una serie de publicaciones de la Asociación Latinoamericana de Zen, cuyo objetivo era la difusión de la doctrina entre un público más general.

Desde ese momento hasta ahora, la oferta de diversos materiales de consulta y aprendizaje producidos en torno a las terapias alternativas y al yoga en especial se amplió considerablemente y diversificó su formato, sumando a los ya existentes, el consumo de numerosos productos resultantes de nuevas tecnologías: videocasetes de enseñanza y práctica de yoga, programas de televisión en cable, revistas dedicadas al yoga y otras terapias alternativas y las dedicadas a la salud y el bienestar en general así como páginas y foros de discusión en Internet. A medida que se amplían los formatos disponibles, se amplían sus modalidades de difusión y el número de personas a las que llega la información.

Los efectos de la globalización a partir de la década del 90 y sus consecuencias en la rapidez y accesibili- 
dad de las comunicaciones no pueden ser obviados a la hora de explicar no sólo el auge, sino también la permanencia del yoga en distintos sectores de la sociedad. Sobre este tema, algunos autores mencionan el impacto de la globalización en los procesos que se han denominado de orientalización de occidente (Campbell, 1997), lo que pareciera haber implicado la apropiación masiva y unilateral de prácticas y teorías de raigambre oriental por parte de individuos occidentales, postura que ha sido criticada en tanto no toma en cuenta la relación dinámica entre Oriente y Occidente (Silva da Silveira, 2005) ${ }^{2}$.

El yoga en Buenos Aires es una versión distinta a la de la India, incluso cuando en su lugar de origen reconozca múltiples versiones y formas de práctica, atravesadas por las distintas visiones propias de cada corriente de pensamiento y por las diferencias que devienen de una sociedad que posee una estructura de castas. Con ello queremos señalar que cada una de las modalidades que pueden hallarse en la Argentina resulta de la situación de contacto entre lo culturalmente adquirido y las nuevas prácticas e ideas asociadas a lo oriental.

La disciplina en cuestión está presente tanto en programas de televisión y revistas de divulgación íntegramente dedicados a mostrar su práctica, como en segmentos televisivos y espacios en Internet donde se aconseja al lector/usuario una serie de métodos y ejercicios propicios para una mejor calidad de vida, pasando por las entrevistas en distintas publicaciones de moda y divulgación realizadas a reconocidos miembros del espectáculo, el cine y la televisión en las que ellos explicitan los beneficios obtenidos a través de su práctica. Todo ello facilita tanto el conocimiento de su existencia como la expansión de un vocabulario que, aunque refigurado, remite a nociones de raigambre oriental.

A continuación, señalaremos brevemente algunos ejemplos de los materiales citados, teniendo especialmente en cuenta aquellos que han sido recurrentemente citados en las entrevistas.

\section{a) Videocasetes}

En los finales de la década del setenta y principios de los ochenta, comenzaron a ofertarse una serie de producciones en videocasete -generalmente provenientes de Estados Unidos- que consistían en la presentación de ejercicios físicos a cargo de un instructor, el que posicionado frente a una cámara, reproducía la modalidad de una clase. El objetivo de este sistema era brindar la posibilidad de realizar la gimnasia en el contexto del hogar, en el horario que al usuario le quedase cómodo, al tiempo que le posibilitaba una guía para realizar la ejercitación de manera segura y efectiva.
Es en este contexto que surgieron los videos de enseñanza de yoga, que se asemejaban con los anteriores en cuanto formato general. Julia, una usuaria de yoga que utilizó este tipo de soporte, nos relataba lo siguiente:

En esa época, allá por los ochentas, tenía un video de yoga para el hogar. Era una instructora de $\mathrm{Ca}-$ lifornia, una rubia muy simpática, de alguna de las escuelas de yoga conocidas allá. Duraba una hora y era como una forma de hacer yoga en tu casa, cuando te venía bien. Lo usé un tiempo y me gustaba, estaba bueno, pero después se volvió aburrido, era hacer todos los días lo mismo... al final me lo sabía de memoria, de tanto repetir... y casi hoy podría repetir la serie completa sin equivocarme, ja, ja, si cierro los ojos la veo a la instructora.

Otros entrevistados comentaron similares cuestiones, concordando en que este sistema ofrecía una guía para la realización de las posturas y contraposturas de manera confortable y en el tiempo y lugar deseado, brindando al usuario un acercamiento a las técnicas del yoga, alejadas del "misticismo" asociado a la disciplina. Como contrapartida, señalaron que se transformaban en un esquema rígido de repeticiones que no contemplaban, por sus propias características, la posibilidad de modificar el orden ni la selección de posturas.

\section{b) Programas de televisión}

Frente al carácter repetitivo de los videos, los programas de televisión brindaban una posibilidad ventajosa. Si bien las clases eran transmitidas siguiendo un formato establecido por el especialista a cargo del programa, la variación entre una y otra sesión televisiva se percibió como una mejoría, en el sentido de poder variar las posturas ejecutadas y acercarse así más a la dinámica de un taller.

Después de eso, las chicas me avisaron que pasaban por cable una clase de yoga todas las mañanas, o casi todas, y creo que la repetían a la tarde. Estaba buena, la hice varias veces. También duraba una hora, la instructora ésta se llamaba Wai Lana, está bueno, creo que todavía sigue, yo hace bastante que no lo veo. (Julia, usuaria)

En el programa de Wai Lana en Infinito hacen yoga (canal de televisión por cable) todos los días lo pasan dos veces, y yo hago a la mañana si puedo, y si no, a la tarde. Después de Wai Lana está el profesor de tai chi chuan, también todos los días. (Alicia, usuaria) 
Alfonsina, una señora de setenta y dos años que vive en la zona norte del área metropolitana, nos cuenta que hizo yoga en un taller de su barrio durante ocho años y que luego abandonó la concurrencia a dichos talleres por la incomodidad que le representaba tener que cumplir horarios. Actualmente realiza la práctica en su casa, siguiendo las instrucciones tanto de Wai Lana como de una instructora de origen japonés en otro canal de cable.

Sí, a Wai Lana la conozco, pero también en Utilísima (canal de televisión por cable) hay una japonesa que da yoga, yo veo los programas de manualidades y después hago yoga.

Quizás uno de los programas que más ha impactado en los usuarios de yoga que entrevistamos es el de la especialista Wai Lana, cuyo ciclo se transmite diariamente desde hace casi diez años por el canal de Cable Infinito, en la franja de 9 a 9.30 horas por la mañana. Rodeada de paisajes solitarios, la especialista presenta su clase ejecutando las asanas e indicando el tipo de respiración que acompañará a cada postura/ contrapostura. Cumple en el desarrollo de cada emisión con todas las instancias comunes a un encuentro de yoga, realizando la preparación, la ejecución de las asanas, la meditación y el cierre ${ }^{3}$. Al respecto, cabe destacar que Wai Lana realiza una guía verbal para llevar adelante la meditación, posibilitando una tutela virtual para su desarrollo.

En la actualidad, son numerosos los programas de cable dedicados tanto a la difusión del yoga como a otras terapias o disciplinas de raigambre oriental. Existen ofertas de yoga, tai chi chuan, ayurveda, feng shui, cromoterapia, entre otras; así como disciplinas occidentales que suman ideas de raigambre oriental, dándole un tinte diferente a sus estilos habituales. Al respecto, y tal como notaran otros autores, la Nueva Era ha producido versiones resignificadas de disciplinas preexistentes, como sucede por ejemplo en el caso de la arquitectura o la decoración de interiores, o la geobiología, las que han sumado a sus pautas tradicionales el uso de nociones y técnicas provenientes de disciplinas orientales, como el Feng Shui.

\section{c) Las revistas de salud y los distintos gra- dos de apropiación de lo oriental}

Como notara Carozzi (2001), a partir de la década del 90, las revistas de divulgación de la Nueva Era mostraron una progresiva revalorización de la ortodo- xia disciplinaria y un progreso énfasis en los objetivos terapéuticos y las credenciales profesionales de muchas de las disciplinas que alentaban. Este hecho fue también observado en otros países, tales como Brasil e Inglaterra (Russo, 1993; Tavares, 1999; Bowman, 1999). Las revistas cambiaron sus nombres, transformándolos en frases evocadoras de la calidad de vida y el cuidado de sí, tal como sucedió con la revista Uno Mismo que cambia su descripción de "revista para el crecimiento personal" a "salud y calidad de vida".

En los últimos años, el número y diversidad de publicaciones quincenales y mensuales que versan sobre la temática de las terapias alternativas ha crecido considerablemente. En cualquier escaparate de revistas de Buenos Aires pueden conseguirse una multitud de publicaciones de este tipo. Si bien todas apuntan a brindar información sobre terapias y calidad de vida, podemos distinguir al menos tres grupos: 1) las que apuntan a difundir diversas disciplinas de la Nueva Era, como "Vivir en Armonía", "Salud Alternativa" y "Terapias Alternativas"; 2) las específicas de una terapia alternativa, como Yoga Integral; 3) las que anuncian y promocionan todas las formas terapéuticas existentes en la oferta del área, que van desde la biomedicina, hasta las religiosas, las tradicionales y las alternativas, como es el caso de "Saber Vivir" y "Buenas Ideas". Estas últimas proponen técnicas que son formas de autotratamiento ${ }^{4}$ basados en las categorías de distintas medicinas. Así, pueden citarse recetas para curar el ojeo, en la página siguiente se recomienda el citado de mantras orientales y la ejecución de posturas del yoga, en la siguiente técnicas de orden biomédico para la sanación de la artritis, en otra el encendido de velas para lograr los favores de algún santo o la limpieza de un ambiente y editoriales que mezclan conceptos de la terapia psicoanalítica con nociones e ideas propias de las terapias alternativas, profundamente refiguradas respecto a sus marcos filosóficos de origen.

Podemos observar una importante diferencia en sus contenidos. Entre las de mayor costo -alrededor de $\$ 8 / \mathrm{U} \$ 3$ - se encuentran las revistas de divulgación de disciplinas específicas, o las que proponen variedad de combinaciones entre la biomedicina y las alternativas, las que hacen especial hincapié en la búsqueda de un bienestar físico, emocional y espiritual, presentando artículos que combinan nociones terapéuticas propias de la psicoterapia y de una espiritualidad asociada a la filosofía oriental. Lo particular de estas publicaciones es la propuesta de una búsqueda de salud en un sentido holístico. La propuesta editorial tiende a fomentar la

3 En un trabajo anterior, notamos que más allá de las diferencias en la duración temporal de los encuentros y de las distintas modalidades posibles de práctica, pueden señalarse cuatro momentos que se hallan presentes siempre: 1) la relajación; 2) la ejecución de posturas y contraposturas corporales y manipulación de la respiración; 3) la meditación; 4) el cierre o agradecimiento (Saizar, 2006).

4 Entendemos por autotratamiento todas aquellas prácticas tendientes a la recuperación del bienestar asociado a la salud que se llevan a cabo en el contexto del hogar o de la comunidad cercana y que no implican la participación directa de un especialista. Como notara Kleinman (1980) el autotratamiento o medicina casera es la primera opción en todas las sociedades más allá de las diferencias étnicas y culturales. 
participación activa de los lectores, publicitando una inmensa oferta de talleres y seminarios a cargo de especialistas extranjeros de reconocida trayectoria en las más variadas disciplinas alternativas. En sus páginas es frecuente hallar avisos invitando a los lectores a participar de encuentros de fin de semana, en los que se dictan seminarios intensivos de técnicas de raigambre oriental, muchas veces publicitados como "retiros espirituales". Lo particular de estas publicaciones es la divulgación de los principios básicos, la oferta de tratamientos y centros de enseñanza de las más variadas terapias alternativas. En lo que se refiere a las ofrecidas, se destacan la recomendación de estrategias de combinación de medicinas alternativas entre sí y la combinación de éstas con la biomedicina.

Entre las revistas de menor costo -el que ronda los $\$ 2 / \mathrm{U} \$ 0,75$ - encontramos una importante variedad de formatos y presentaciones. En general, incluyen consejos y recomendaciones para el cuidado de la salud a partir de nociones propias de la medicina tradicional, como la herboristería y las técnicas para curar taxa tradicionales -entre los que se incluyen el mal de ojo, los nervios o la culebrilla-, con nociones biomédicas que apuntan sobre todo a las prácticas de prevención de la enfermedad y en menor medida, también se realizan propuestas terapéuticas del espectro alternativo. El elemento característico de estas publicaciones es que brindan información sobre una gama variada de medidas de autotratamiento, es decir, sus contenidos reflejan las distintas vías por las cuales los individuos pueden recuperar su salud en el contexto del hogar y sin mediación de un especialista. A modo de ejemplo, en "Buenas Ideas" (Nro 9, Año 1) se promocionan las "incré́bles propiedades" de la infusión de manzanilla, la que permite tratar asma, molestias de la menopausia, insomnio, gastritis y nerviosismo, al tiempo que previene la aparición de reumatismo, alergias y gripe; en otra página brindan consejos de autoayuda para ser feliz, los zapatos indicados para cada tipo de signo del zodíaco, las propiedades de frutas, vegetales y hierbas para la cosmética y consejos del escritor Paulo Coelho para el crecimiento espiritual. Finalmente, en este rango de publicaciones se destacan -en cuanto a cantidad- las recomendaciones que hacen referencia a los principios de la medicina tradicional y en segundo lugar a las religiosas de raigambre católica y a las alternativas.

\section{d) Páginas y foros de discusión en Internet}

El consumo de contenidos informáticos relativos a las terapias alternativas se acrecentó a medida que la tecnología de Internet fue expandiéndose y su uso se transformó en algo cotidiano, presente en la mayoría de los hogares de las llamadas clases medias y altas. Las propuestas se ampliaron y diversificaron a través del tiempo y hoy en día, pueden hallarse una importante variedad de formatos y opciones. Como parte del trabajo de campo que realizáramos indagando en las ofertas posibles del yoga -no sólo como opción terapéutica sino como propuesta de estilo de vida o marco filosófico-, encontramos ofertas que varían sus estilos y contenidos y parecieran destinarse a distintos tipos de consumidores.

Por un lado, existen portales exclusivos de pequeños institutos de Yoga, centros de enseñanza terciarios o universidades, que generalmente estructuran su diseño relatando la historia institucional, las bases filosóficas que fundamentan el tipo de yoga propuesto, las actividades sugeridas y una breve descripción curricular de los docentes. Entre las distintas páginas que relevamos (www.akirelax.com; www.terra.com/ foros/yoga; www.wailana.com; www.yogabuenosaires.com ${ }^{5}$ ), es especialmente interesante, por sus contenidos, el sitio de Uni-Yoga, dirigida en la Argentina por el Profesor Edgardo Caramella y fundada por el Profesor DeRose, de origen brasileño. En este extenso sitio se ofrece, más allá de los contenidos similares a otras páginas, la posibilidad de realizar descargas gratuitas de material bibliográfico, observar videos de la práctica, recorrer galerías de fotos y obtener contactos con otros sitios similares. En un apartado especial se enlistan las distintas entidades que han adoptado el Programa de Yoga en Empresas, cuyo objetivo es "mejorar la calidad de vida de los trabajadores, generar crecimiento de la productividad y reducir costos por enfermedades de trabajo y ausentismo". Entre las nombradas, figuran tanto reconocidas empresas multinacionales y nacionales como entidades de la administración pública. Al pie de esta lista figuran una serie de entidades educativas con las que poseen convenios para dictar sus talleres.

Por otra parte, existe una amplia oferta de Foros en que los usuarios registrados tienen la posibilidad de intercambiar ideas, experiencias y conceptos sobre la práctica del yoga. Algunos de estos sitios están especializados en la discusión acerca de una variante de la disciplina en particular o de una rama filosófica, pero en general las contribuciones individuales son heterogéneas, pueden incluso implicar el anexo al temario en discusión de otras terapias alternativas, tales como reiki, bioenergética, flores de Bach o reflexología. Estos espacios virtuales cuentan algunas veces con un moderador que responde algunas preguntas e intermedia entre los participantes, pero en su mayoría las respuestas son brindadas por otros usuarios, quienes han realizado ya un tipo de práctica -yoga u otra disciplina alternativa- o poseen conocimientos que podríamos denominar filosóficos. El tipo de preguntas más frecuentes responde a criterios generales sobre la disciplina, tales como “¿Qué beneficios trae el yoga?”, “¿Dónde pue- 
do practicarlo?”, “¿Cuántas veces por semana debería practicar para que me haga bien?” Las respuestas más frecuentes son -en el mismo orden- "Es bueno para sentirte mejor en todos los aspectos... te hace bien para el stress... te ayuda a adelgazar... te relaja", "Voy a un centro que está cerca de mi trabajo, fijate porque hay varias sucursales... Averigua en tu barrio, en el mío hay centros gratis de la municipalidad", "Dicen que es bueno al menos dos veces por semana...Yo voy una y me hace bien...Tendrías que hacerlo todos los días para notar cambios positivos", etc.

De acuerdo a los entrevistados, el relato de otros usuarios y la evaluación que ellos hacen de su experiencia es un factor a considerar al momento de elegir o rechazar un taller, un especialista o un tipo de práctica alternativa.

\section{e) Libros}

En la década del sesenta comenzaron a aparecer en las librerías comerciales distintas obras destinadas a la divulgación del yoga, tanto en su aspecto teórico como en el práctico. Esta serie de libros tenía como objetivo presentarlo como una ejercitación corporal que alentaba la relajación del cuerpo y de la mente, pero sobre todo, que posibilitaba el aprendizaje y adquisición de un modo de respiración y un estilo de vida tendiente a preservar el equilibrio entre el interior del hombre y su entorno. A modo de ejemplo, el libro de Philippe de Meric, $A B C$ del Yoga (1962), prometía acercar al hombre urbano una técnica consistente en una serie de ejercicios corporales "fáciles de hacer", apuntaba una serie de pasos para lograr el desarrollo de una respiración "amplia y correcta"; el libro Yoga para todos, de Desmond Dunne (1963), se promocionaba "como una novela fácil de leer, y que significa para todos salud, bienestar y un desarrollo total y armónico. Una obra que adapta esta suma de sabiduría oriental de todos los tiempos a las necesidades del Occidente de hoy. Un método ideal de cultura física y mental para todos: hombres y mujeres de cualquier edad"; o el libro Yoga para la mujer, de Edouard Longue (1962), presentado como "una maravillosa selección de ejercicios físicos y mentales para las mujeres. Yoga para su salud, su atractivo y su alegría”.

Hoy en día, la oferta de libros de divulgación de estas prácticas orientales se ha ampliado considerablemente, incluyendo obras de autores variados en cuanto a su formación y sus propuestas. Por su alto nivel de impacto entre los usuarios de las terapias alternativas, se destacan autores como Osho, Deepak Chopra, Paulo Coelho y Bárbara Brennan. A diferencia de las primeras publicaciones, que se atenían a la descripción de las técnicas de respiración y manejo del cuerpo, las actuales hacen mayor hincapié en el aspecto "espiritual" de estas prácticas, manteniendo la promoción de los aspectos relacionados a la salud y a la calidad de vida, además de la posibilidad del auto-conocimiento como vía de bienestar y salud.
Estos autores son citados por los entrevistados para referirse de una manera concreta a distintas cuestiones, desde brindar conceptos sobre la cantidad y calidad de chakras, la consistencia del aura, la raíz y devenir del karma, hasta nuevas formas de meditación y técnicas utilizadas para generar nuevas estrategias de auto-tratamiento, tales como colorear mandalas, visualizar cambios en el aura, etc.

Mónica, especialista de yoga, reiki y Feng Shui, nos decía:

Yo sigo a Chopra, él dice que el karma son las asignaturas pendientes de vidas pasadas. El poder del karma postula la ley de causa y efecto, esto quiere decir que todo cuanto te ocurre, el efecto, tuvo una causa anterior. El funcionamiento de la ley kármica te dice que vos podés ser dueño de tu propio destino. Las emociones de la vida, reflejan lo que vos no tenés, lo que es débil en vos, y es eso que te dificulta el triunfo. Además, esas dificultades te permiten entender, porque te explican las experiencias o obligaciones que evitaste en alguna vida pasada. De una manera simple, el karma es tropezar siempre con la misma piedra.

Al respecto, es interesante destacar que los distintos autores de consumo frecuente entre los usuarios de terapias alternativas proponen la apropiación de los conceptos filosóficos orientales como un medio de obtener no sólo una mayor calidad de vida, sino fundamentalmente, de lograr la concreción de los deseos individuales, entendida como el éxito a la manera occidental, es decir, una sumatoria de bienestar anímico, físico, vincular y económico.

\section{f) La oferta gratuita}

Consideramos una importante vía de difusión y conocimiento el hecho de que el yoga se transformara, desde la década del 80 y con mayor impulso en la década del 90, en una oferta gratuita en centros barriales, gimnasios, talleres de las distintas municipalidades del conurbano bonaerense y numerosas parroquias católicas, donde la ofrecían y ofrecen como una actividad gratuita para sus fieles y miembros de la comunidad interesados en participar de las mismas.

Los gobiernos municipales del conurbano, a través de sus centros culturales ubicados en las localidades de cada partido, ofertan a los vecinos una serie de talleres, entre las que se incluyen yoga, gimnasia, cine debate, ajedrez, danzas folklóricas, etc. El único requisito para participar en ellos es demostrar la pertenencia al partido. En algunos casos, se solicita el pago de una cuota de colaboración voluntaria, la que en el 2007 rondaba los \$10 (US 3 aproximadamente), mientras que en la mayoría la oferta es absolutamente gratuita.

Los talleres de yoga en las iglesias del área Metropolitana se ofrecen en un contexto que mixtura una 
gran diversidad de ofertas, entre las que se incluyen desde talleres de pintura sobre telas y cursos de cocina a catecismo para adultos, que forman parte de una estrategia de apertura de la iglesia hacia la comunidad en la que se inserta. La participación en ellos es absolutamente gratuita. También es interesante destacar que a partir de la década del 90 comenzaron a ofrecerse talleres de yoga a los grupos de sin techo (deambulantes tanto hombres como mujeres adultos) que concurrían a los comedores parroquiales, como un medio de que realizaran una actividad física que no demandara una especial destreza física y que les posibilitara interactuar en grupos de pares, en el marco de una serie de acciones tendientes a reestablecer -en parte- la dificultad de establecer vínculos afectivos, situación que por su aislamiento familiar es característica de los homeless ${ }^{6}$.

En ese mismo período comenzó a ofrecerse en distintos gimnasios del conurbano y de la capital la posibilidad de realizar yoga en un espacio habitualmente dedicado a prácticas corporales occidentales, como aerobics, step, gimnasia localizada o entrenamiento en resistencia física. Lo particular de esta oferta, según hemos observado, es que los usuarios no la utilizan de modo permanente, sino que "prueban" unas pocas clases y luego abandonan. Son, podríamos decir, usuarios en tránsito.

\section{g) La recomendación del yoga por parte de los biomédicos}

Si bien los usuarios del yoga no muestran especial interés en la opinión que los biomédicos puedan verter sobre la disciplina, es interesante considerar el fenómeno de la aceptación que ella obtuvo en ciertos ámbitos, tales como gerontología, traumatología, clínica y obstetricia, por parte de algunos biomédicos, quienes comenzaron a derivarla a mujeres embarazadas para realizar sus cursos de pre-parto, a pacientes que padecen de artritis, de problemas de columna, de diabetes, de parkinson y de enfermedades crónicas que implican, en su mayoría, sufrimiento y dolor. Desde la perspectiva de los especialistas biomédicos, la mencionada disciplina se percibe como un ejercicio tranquilo, que no requiere esfuerzos físicos y que, por ende, puede ser practicada por individuos de las más disímiles edades y estados físicos. En tanto no implica el consumo de fármacos de ninguna clase, su práctica no genera contradicciones con la propia prescripción terapéutica.

En el relato de nuestros entrevistados, dicha recomendación aparece frecuentemente como un motivo de inicio en la disciplina, si bien los conceptos que un usuario de este tipo puede expresar al cabo de un tiempo son ajenos a los marcos explicativos de las teorías biomédicas. En otras palabras, probablemente se refiera a la eficacia del yoga en términos tales como energía, armonía de los chakras, relajación y meditación, y no en cuanto a las bondades de una "gimnasia suave y no invasiva".

Por otra parte, es cada vez más frecuente que los psicoterapeutas -en su mayoría pertenecientes a las escuelas cognitiva y conductual- recomienden la práctica del yoga a los pacientes como estrategia de complementariedad terapéutica. Vale decir, él se suma al tratamiento psicoterapéutico que ofrece el especialista. Como notáramos en un trabajo anterior (Korman y Saizar, 2006) la mayoría de los psicoterapeutas cognitivos presentan una idea del yoga acorde a las técnicas de relajación, respiración y meditación. Desde la perspectiva de estos terapeutas, el yoga permite acrecentar las habilidades del paciente en lo que hace a las técnicas de relajación, a la vez que, en su vida, implica un espacio enteramente dedicado a la relajación y a una práctica de autoconocimiento que generan efectos positivos en conjunción con la terapia biomédica. La valoración positiva de las técnicas de relajación, propias de la terapia cognitiva conductual, la propia práctica del psicoterapeuta, la recomendación de pares y los efectos positivos dan lugar a la recomendación de complementariedad.

Respecto al relato de los usuarios del yoga, cuando su práctica se da en combinación con un tratamiento de índole psicoterapéutico, las mejoras y ventajas de la complementariedad se analizan en términos diferentes. Por un lado, se refiere una mejor circulación de energía, una armonización de los chakras, el logro del desapego, entre otros. Por el otro, se comprende que ambas disciplinas actúan en niveles distintos de la persona, y que mientras la psicoterapia actúa a partir de la palabra, el yoga basa su eficacia en el movimiento corporal y energético.

\section{Conclusión}

Los motivos de la amplia divulgación de las terapias alternativas y del yoga en particular, pueden atribuirse no sólo a causas externas, tales como la visibilidad notoria de la disciplina a través de su difusión en medios masivos de comunicación, la diversidad de ofertas y de costos o la posible recomendación de especialistas biomédicos, sino fundamentalmente a la posibilidad de apropiación y refiguración de las nociones propias de estas disciplinas de raigambre oriental y a la eficacia terapéutica atribuida a la práctica por los mismos usuarios.

En este sentido cabe destacar que el yoga puede ser fácilmente refigurado y entendido en términos de la religiosidad del individuo, mayoritariamente católica, o 
de las prácticas tradicionales del curanderismo en lo que hace a individuos de los sectores llamados populares. El yoga se constituye en una práctica de ingreso al ámbito de las terapias alternativas, por la que los individuos transitan o permanecen mientras amplían su campo de experiencias de búsqueda de salud y calidad de vida.

Como hemos visto en el desarrollo del presente trabajo, el yoga pasó a formar parte de las ofertas te- rapéuticas y de las actividades destinadas a lograr una mejor calidad de vida, transformando sus principios filosóficos y técnicas específicas en una práctica posible para cualquier individuo que quisiese mejorar sus condiciones de existencia, convirtiéndose, hoy en día, en una práctica de consumo adoptada por diferentes individuos, con disímiles niveles de instrucción e ingreso económico, de la que, finalmente, "todos saben algo".

\section{Referencias}

ALBANESE, Catherine. Nature religion in América: from the Algonkian indians to the New Age. Chicago and London: The University of Chicago Press, 1990.

The magical staff: quantum healing in the New Age. En: LEWIS, J. y MELTON, J. (Comp.) Perspectives in the New Age. Albany: State University of New York Press, 1992. p. 68-84.

BARROSO, María. A construção da pessoa "oriental" no Ocidente: um estudo de caso sobre o Siddha Yoga, 1999. Dissertação (Mestrado em Antropologia Social). Rio de Janeiro: PPGAS/MN/UFRJ, 1999.

BOWMAN, Marion. Healing in the spiritual marketplace: consumers, courses and credentialism. Social Compass, 46 (2), 1999.

CAMPBELL, Colin. A orientalização do Ocidente. Religião e Sociedade. Rio de Janeiro: Record, 1997.

CARINI, Catón. El Budismo Zen en la Argentina. 4to Congreso Virtual de Antropología y Arqueología, 2004.

CAROZZI, María Julia. Nova Era: a autonomia como religião. En: CAROZZI, M. (Comp.). A Nova Era no Mercosul. Petrópolis: Vozes, 1999. p. 149-190.

Nueva Era y Terapias Alternativas. Construyendo significados en el discurso y la interacción. Buenos Aires: Ediciones de la Universidad Católica Argentina, 2001.

DOUGLAS, Mary. Estilos de Pensar. Barcelona: Gedisa, 1998.

DUNNE, Desmond. Yoga para todos. Barcelona: Ediciones Toray, 1963.

ELIADE, Mircea. Técnicas del Yoga. Buenos Aires: Cairos, 1999.

IDOYAGA MOLINA, Anatilde. Ethnomedicine and world-view. A comparative analysis of the rejection and incorporation of the contraceptive methods among argentine women. Anthropology and Medicine, 4 (2), 1997.

Culturas, enfermedades y medicinas. Reflexiones sobre la atención de la salud en contextos interculturales de Argentina. Buenos Aires: CAEA-CONICET, 2002.

KLEINMAN, Arthur. Patients and healers in the context of culture. Berkeley: University of California Press, 1980.

KORMAN, Guido y SAIZAR, Mercedes. Psicoterapia y Yoga. La inclusión del yoga como terapia complementaria en los tratamientos psicoterapéuticos cognitivos en Buenos Aires (Argentina). Revista Brasilera de Sociología de las Emociones, v. 13, 2006.
LAPLANTINE, François. Antropología de la Enfermedad. Buenos Aires: Ediciones del Sol, 1999.

LONGUE, Edouard. Yoga para la mujer. Barcelona: Ediciones Toray, 1962.

MASSON OURSEL, Peter. El Yoga. Buenos Aires: Eudeba, 1962.

MC GUIRE, Meredith y KANTOR, Deborah. Ritual Healing in Suburban America. New Brunswick: Rutgers University Press, 1998.

MERIC, Philippe. ABC del Yoga. Barcelona: Ediciones Toray, 1962.

ORGANIZACIÓN MUNDIAL DE LA SALUD. Estrategias para la Medicina Tradicional 2001-2005. Genova: Organización Mundial de la Salud, 2002.

PITLUK, Roberto. El shiatsu. Efectos terapéuticos e impacto cosmovisional en pacientes urbanos. Scripta Ethnologica, XXIV, 2002.

QUILES, Ismael. Que es el Yoga. Buenos Aires: Editorial De Palma Russo, 1987.

REVISTA BUENAS IDEAS, nro. 9, año 1, 13 de abril de 2006. ISSN 1669-791X.

RUSSO, Jane. O corpo contra a palavra. As terapias corporais no campo psicológico dos anos 80 . Rio de Janeiro: UFRJ, 1993.

SAIZAR, Mercedes. Los Sin Techo: La mirada de los actores. Regiones y Desarrollo Sustentable, 4. México: El Colegio de Tlaxcala, 2003.

Nuevos caminos en la búsqueda de salud. El yoga en el Área Metropolitana. Buenos Aires: CAEA, 2005.

Yoga y complementariedad terapéutica, 2006. Tesis (Doctorado en Cultura y Sociedad). Buenos Aires: IUNA/CAEA, 2006.

SILVA DA SILVEIRA, Marcos. New Age \& Neo-Hinduismo: uma via de mão dupla nas relações culturais entre Ocidente e Oriente. Ciencias Sociales y Religión, 7, 2005.

TAVARES, Fátima R. Holismo terapéutico no ambito do movimiento Nova Era no Rio Janeiro. En: CAROZZI, M. (Org.). A Nova Era no Mercosul. Petrópolis: Vozes, 1999. p. 80-105.

TOLA, Fernando y DRAGONETTI, Carmen. La Filosofía Yoga. Buenos Aires: Cairos, 2006.

ZIMMER, Henry. Filosofías de la India. Buenos Aires: Eudeba, 1973. 


\title{
Everybody knows. Dissemination and appropriation of yoga techniques in Buenos Aires (Argentina)
}

\begin{abstract}
In this article, the author presents Yoga as a way to reach and initiate not only spiritual growth, but also the health notion that is present in the New Age movement. The article analyzes the appropriation and reconfiguration processes of the eastern discipline, as well as its beginning and dissemination considering the participants' perspective - users and specialists. It also reflects about the material from different means of diffusion as magazines, books, exercise videos, TV shows and internet discussions about the subject.
\end{abstract}

Key-words: diffusion, appropriation, yoga, Buenos Aires, Argentina.

\section{Todo mundo sabe. Difusão e apropriação das técnicas de ioga em Buenos Aires (Argentina)}

\section{Resumo}

Neste trabalho, a autora apresenta o ioga como uma das práticas que permitem o acesso e a iniciação a outras buscas espirituais e da saúde próprias do movimento da Nova Era. Analisa o processo de apropriação e refiguração das noções próprias da disciplina oriental, sua aparição e difusão, considerando a perspectiva dos praticantes -usuários e especialistas- e o material proveniente de vários meios de divulgação em massa, tais como revistas, livros, vídeos de prática, programas de televisão e discussões na Internet, dedicados ao tema.

Palavras-chave: difusão; apropriação; ioga; Buenos Aires; Argentina.

Data de recebimento do artigo: 17 de outubro de 2008

Data de aprovação do artigo: 25 de março de 2008 